\title{
Toxicomanie d'un anesthésiste au fentanyl : la preuve formelle par analyse des cheveux en utilisant la CPG/SM/SM
}

\section{Evidence of fentanyl abuse by an anesthetist through hair analysis using GC/MS/MS}

Marion VILLAIN, Vincent CIRIMELE, Bertrand LUDES, Pascal KINTZ*

Institut de Médecine Légale, 11, rue Humann - 67000 STRASBOURG - France

*Auteur à qui adresser la correspondance : Docteur Pascal KINTZ

Tél : 0390243349 - Fax : 0390243362 - e-mail : pascal.kintz@wanadoo.fr

(Reçu le 24 octobre 2000 ; accepté le 8 janvier 2001)

\section{$R \hat{E} S U M E ́$}

Un médecin anesthésiste exerçant en Suisse est soupçonné d'usage détourné de fentanyl. Afin de palier aux limitations des analyses urinaires, négatives pour le fentanyl à plusieurs reprises, une recherche au niveau capillaire a été réalisée, pouvant déterminer une éventuelle exposition répétée à ce médicament. Pour cela, une méthode par chromatographie gazeuse couplée à un spectromètre de masse tandem a été mise au point, comprenant une incubation de la poudre de cheveux dans du tampon Soerensen et une purification sur colonne Isolute CI8.

Ce procédé validé (linéaire entre 20 et $1000 \mathrm{pg} / \mathrm{mg}$, limite de détection de $20 \mathrm{pg} / \mathrm{mg}$ et rendement d'extraction de $82 \%$ pour une concentration de $100 \mathrm{pg} / \mathrm{mg}$ ), a permis de doser le fentanyl à la concentration de $644 \mathrm{pg} / \mathrm{mg}$. Celle ci est très importante en regard des concentrations retrouvées dans la littérature et signifie que ce médecin consomme de façon régulière ce stupéfiant.

\section{MOTS-CLÉS}

fentanyl, abus chronique, cheveux, CPG/SM/SM.

\section{SUMMARY}

An anesthetist, working in Switzerland, was suspected of fentanyl misuse. His urine analyses, biological fluid usually employed to document drug consumption, were always found negative. However it is known that the elimination of such drugs occurs within a few days. In contrast, hair allows a drug administration to be tracked back for months or even years. This is the reason why a method using a GC/MS/MS system was developed to detect fentanyl in hair.

After the validation of the procedure (linearity between 20 and $1000 \mathrm{pg} / \mathrm{mg}$, limit of detection of $20 \mathrm{pg} / \mathrm{mg}$ and extraction recovery of $82 \%$ at $100 \mathrm{pg} / \mathrm{mg}$ ), we were able to detect fentanyl at a concentration of $644 \mathrm{pg} / \mathrm{mg}$. This result is the proof of chronic abuse of fentanyl by the anesthetist.
KEY-WORDS

fentanyl, chronic abuse, hair, GC/MS/MS. 


\section{Introduction}

Le fentanyl, N-(1-phényléthyl-4-pipéridyl)-propionanilide, est un analgésique central réservé à l'anesthésie de courte, moyenne ou longue durée. Il est utilisé dans les protocoles de neuroleptanalgésie, d'anesthésie générale balancée et d'anesthésie analgésique à doses élevées.

C'est un morphinomimétique très puissant qui provoque une anesthésie chirurgicale environ 100 fois supérieure à celle de la morphine chez l'Homme.

Comme les autres analgésiques, le fentanyl peut faire l'objet d'abus, en particulier dans le milieu médical où l'accès y est facilité $(1,2,3)$. Le fentanyl et ses analogues ont également été identifiés comme drogues de la rue, impliquant un traffic ou encore leur production clandestine : c'est l'exemple de l'alpha-methylfentanyl, baptisé "China White".

Habituellement sous forme de solution injectable, le fentanyl est commercialisé, depuis quelques années, sous la forme de patches transdermiques, souvent prescrits pour le traitement de douleurs chroniques (4).

Généralement, le dosage du fentanyl est réalisé dans le sang et les urines mais sa durée de détection est limitée de part sa disparition rapide des fluides corporelles. En effet, le fentanyl a une durée de vie de 3 à 12 heures : une injection en intraveineuse de fentanyl de $2 \mu \mathrm{g} / \mathrm{kg}$ conduit à une concentration de $11 \mu \mathrm{g} / \mathrm{L}$ et celle ci décroit jusqu'à $1 \mu \mathrm{g} / \mathrm{L}$ en 1 heure.

En ce qui concerne son métabolisme, plus de $85 \%$ du fentanyl ingéré est excrété dans les urines sous 3-4 jours.

L'analyse des cheveux montre son interêt en augmentant la fenêtre de détection de quelques jours à plusieurs mois (selon la longueur des cheveux analysés). En effet, outre le fait que les cheveux sont plus faciles à prélever et à conserver que le sang ou les urines, ce type de prélevement présente l'avantage unique d'être le reflet d'une exposition répétée. Cela peut représenter un complément idéal des résultats de dosages urinaires conventionnels, les analyses urinaires pouvant rendre compte d'une exposition récente d'un individu à une drogue et les analyses capillaires pouvant mettre en évidence un abus à long terme.

La revue de la littérature, pauvre concernant ce sujet, montre cependant l'existence de trois travaux relatant le dosage du fentanyl dans les cheveux : Wang et al (5), utilisant des techniques immunologiques, est confronté à des problèmes de réactions croisées et donc de faux positifs. Les deux autres $(6,7)$, basées sur des techniques chromatographiques, dosent des concentations de 20 et 100 picogrammes de fentanyl par milligramme de cheveux. Ces concentrations étant très faibles, il a été nécessaire de développer une methode sensible et très spécifique afin de pouvoir doser des concentrations de l'ordre du picogramme.

Le cas rapporté ici est celui d'un médecin fortement soupconné d'un abus chronique de fentanyl, et pour lequel une analyse au niveau des cheveux a été demandée par un confrère suisse.

\section{Historique}

Un médecin anesthésiste d'une cinquantaine d'années, pratiquant dans une clinique du canton de Genève, est suspecté de consommer du fentanyl de manière illicite. Il a été convoqué à plusieurs reprises dant le but de réaliser des analyses urinaires. Celles ci se sont toujours révélées négatives, mais la molécule n'est détectable dans les urines que quelques jours après la dernière prise. Par ailleurs, le rendez-vous ayant toujours été pris plusieurs jours avant, il pouvait être aisé au praticien de s'abstenir sur une courte durée.

De manière à augmenter la fenêtre de détection, il semblait nécessaire de réaliser des analyses capillaires. En effet, selon la longueur des cheveux, il est possible de doser des molécules ingérées jusqu'à plusieurs mois avant le moment du prélèvement.

Dans le cas présenté, une mèche de cheveux de trois centimètres a été prélevée au niveau du vertex postérieur et envoyée, pour analyse, à l'Institut de Médecine Légale de Strasbourg.

\section{Matériel et méthodes}

Après décontamination de la mèche de cheveux par le dichlorométhane ( 2 fois $5 \mathrm{ml}, 2 \mathrm{~min}$ ), l'échantillon de cheveux est pulvérisé dans un broyeur à boulet jusqu'à l'obtention d'une poudre fine. $80 \mathrm{mg}$ sont incubés une nuit dans $2 \mathrm{ml}$ de tampon Soerensen $\mathrm{pH} 7,6$, en présence de 2,5 ng de fentanyl- $\mathrm{d}_{5}$ (promochem) utilisé comme standard interne, à $40^{\circ} \mathrm{C}$ puis centrifugés. Le résidu est repris dans $1 \mathrm{ml}$ de tampon frais, agité et centrifugé. Les phases aqueuses réunies sont extraites en phase solide. La colonne Isolute $\mathrm{C} 18$ (Touzart et Matignon) est activée par $3 \mathrm{ml}$ de méthanol puis conditionnée par $3 \mathrm{ml}$ d'eau bidistillée. L'échantillon est déposé et la colonne est rincée par 2 fois $1 \mathrm{ml}$ d'eau bidistillée. Après séchage de la colonne, 1 heure, sous vide, le fentanyl est élué avec 3 fois $0.5 \mathrm{ml}$ d'acétone/dichlorométhane $(3 / 1, v / v)$. La phase organique est évaporée sous vide et le résidu repris dans $30 \mu \mathrm{lde}$ méthanol.

Le système d'analyse consiste en un passeur automatique Finnigan A200S et un chromatographe "Varian 
3400" couplé à un spectromètre de masse tandem "Finnigan TSQ700" (GC/MS/MS).

Un aliquot de $2 \mu 1$ est injecté en mode splitless (injecteur : $250^{\circ} \mathrm{C}$ ) dans une colonne HP5-MS (30 $\mathrm{mx}$ $0,25 \mathrm{~mm} \times 0,25 \mathrm{um}$ ), traversée par de l'hélium N55 à un débit de $1 \mathrm{ml} / \mathrm{min}$. Le four est programmé à une température initiale de $60^{\circ} \mathrm{C}$, puis chauffé à $30^{\circ} \mathrm{C} / \mathrm{min}$ jusqu'à atteindre une température de $295^{\circ} \mathrm{C}$, maintenue pendant $5 \mathrm{~min}$. La durée totale de l'analyse est de 14 min.

Le détecteur de masse est utilisé en mode impact électronique avec une énergie d'ionisation de $70 \mathrm{eV}$ et une température de source de $150^{\circ} \mathrm{C}$. L'acquisition est réalisée en mode SRM (selected reaction monitoring).

L'ion majoritaire obtenu en EI/Q1/MS est selectionné comme ion parent $(\mathrm{m} / \mathrm{z} 245)$ et les ions fils $(\mathrm{m} / \mathrm{z} 245$, $189,146)$ sont détectés dans le troisième quadripôle (figure 1), après la collision avec l'argon (chambre de collision : 0,2 mTorr, énergie de collision : $-10 \mathrm{eV}$ )

Le fentanyl est identifié à partir de son temps de rétention et de l'abondance des trois ions de confirmation $(R t=11,91 ; \mathrm{m} / z \quad 245,189,146)$.

La quantification est faite par rapport au fentanyl- $\mathrm{d}_{5}$ $(R t=11,89 ; m / z \quad 250)$.

La linéarité est réalisée à partir de $50 \mathrm{mg}$ de poudre de cheveux témoins (négatifs pour le fentanyl) surchargés avec $1(20 \mathrm{pg} / \mathrm{mg}), 2,5(50 \mathrm{pg} / \mathrm{mg}), 5(100 \mathrm{pg} / \mathrm{mg})$, $25(500 \mathrm{pg} / \mathrm{mg})$, et $50(1000 \mathrm{pg} / \mathrm{mg}) \mathrm{ng}$ de fentanyl.

Le rendement d'extraction est déterminé en comparant les surfaces de pics du fentanyl extrait de $50 \mathrm{mg}$ de poudre de cheveux témoins surchargés pour une concentration finale de $100 \mathrm{pg} / \mathrm{mg}$ avec la surface de pic d'une solution standard à la même concentration.

La limite de détection est évaluée à l'aide de concentrations décroissantes de fentanyl, jusqu'à l'obtention d'une réponse correspondant à 3 fois le bruit de fond.

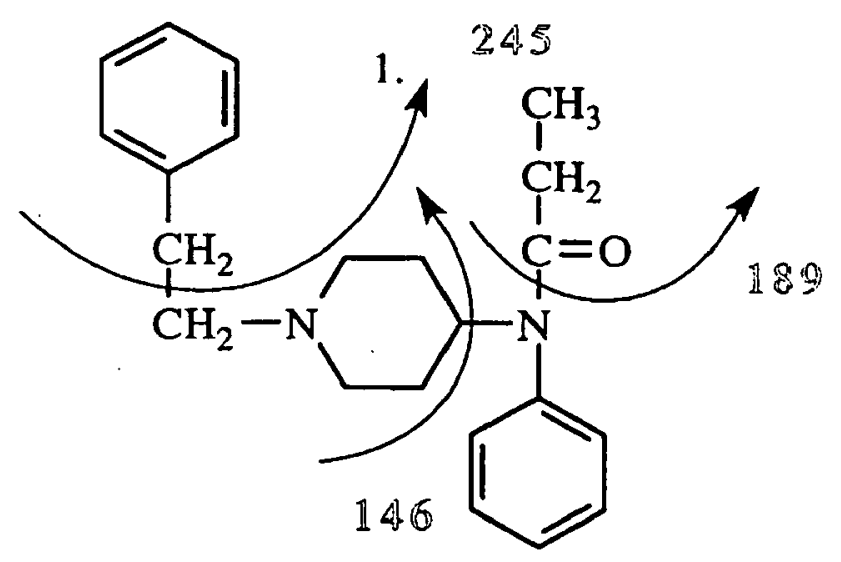

$\mathrm{C}_{22} \mathrm{H}_{28} \mathrm{~N}_{2} \mathrm{O}$

$\mathrm{MM}=336,47$

Figure 1 : Schéma réactionnel de fragmentation du fentanyl.

\section{Résultats et discussion}

Dans un premier temps, nous avons optimisé le procédé d'extraction du fentanyl de la matrice capillaire. En effet, une interférence au niveau de notre pic de fentanyl était visible lors d'une extraction liquide-liquide par les solvants chloroforme/isopropanol/n-heptane $(25 / 10 / 65, \mathrm{v} / \mathrm{v} / \mathrm{v})$, à $\mathrm{pH} 7,6$ (tampon Soerensen), montrant la coélution d'une molécule endogène présente dans les cheveux. Nous avons résolu ce problème en procédant à une extraction en phase solide sur cartouche Isolute $\mathrm{C} 18$.

Dans les conditions chromatographiques utilisées, le pic de fentanyl est parfaitement isolé.

L'analyse par CPG/SM/SM des cheveux montre un comportement linéaire pour des concentrations allant de 20 à $1000 \mathrm{pg} / \mathrm{mg}$, avec un coefficient de corrélation de 0,999 . La courbe de calibration est représentée figure 2 et correspond à la regression linéaire entre le rapport des surfaces de pic (fentanyl / fentanyl- $\mathrm{d}_{5}$ ) et les concentrations finales en fentanyl. L'équation de cette droite est $y=288,809 x-5,292$.

La limite de détection du fentanyl, déterminée pour un rapport signal sur bruit de fond de 3 , est de $20 \mathrm{pg} / \mathrm{mg}$.

Pour une concentration finale en fentanyl de $100 \mathrm{pg} / \mathrm{mg}$, le rendement d'extraction est de $82 \%$.

L'analyse de l'échantillon de cheveux met en évidence le fentanyl à une concentration de $644 \mathrm{pg} / \mathrm{mg}$ (cf figure 3). Étant donné que les cheveux poussent à la vitesse d' $1 \mathrm{~cm}$ environ par mois et que la mèche mesure 3 $\mathrm{cm}$, cette concentration représente la consommation moyenne de l'individu sur une période de trois mois.

Cette concentration est supérieure à celles décrites dans les deux cas précédents, $20 \mathrm{pg} / \mathrm{mg}$ (6) et $100 \mathrm{pg} / \mathrm{mg}$ (7), mais reste particulièrement faible comparée à celle des autres xénobiotiques dosés dans les cheveux.

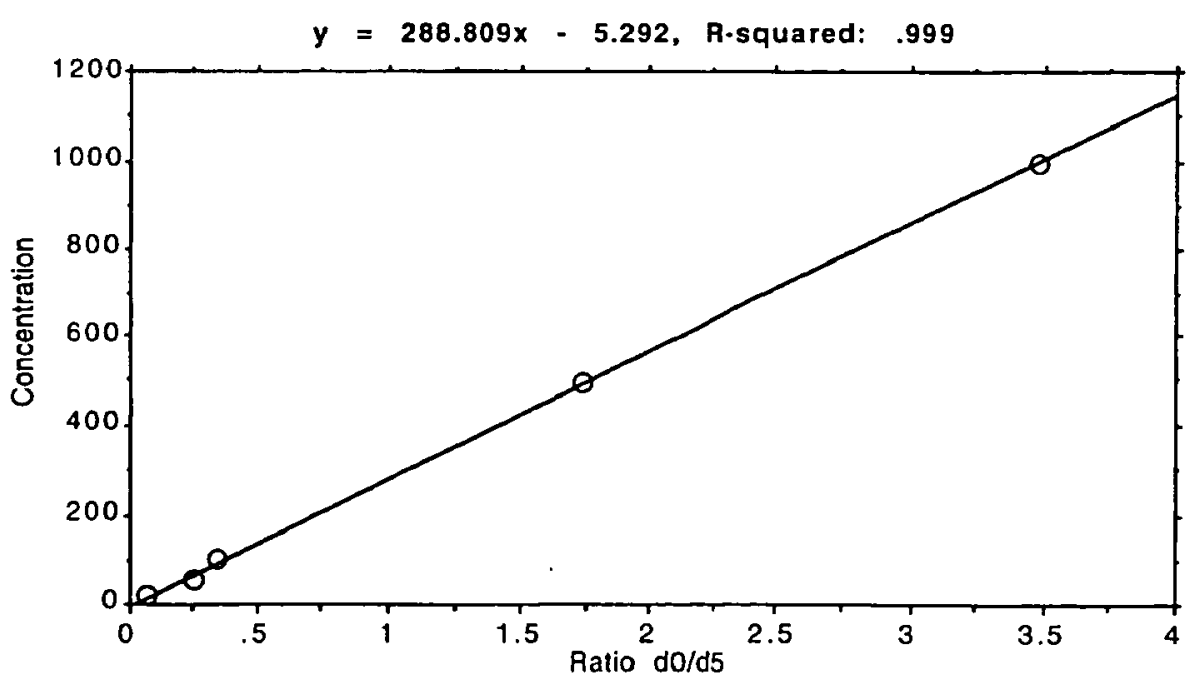

Figure 2 : Courbe de calibration pour des concentrations de fentanyl de 20 à $1000 \mathrm{pg} / \mathrm{mg}(y=288,809 x-5,292$; $r^{2}=0,999$ ). 
Annales de Toxicologie Analytique, vol. XIII, $n^{\circ}$ 1, 2001

D:Ifentanyllfent3110i echantillon( $80 \mathrm{mg}$ ), eimsms-SRM-LR2-argon0,2

10/26/00 01:46:19 AM

RT: $10.98-12.92$
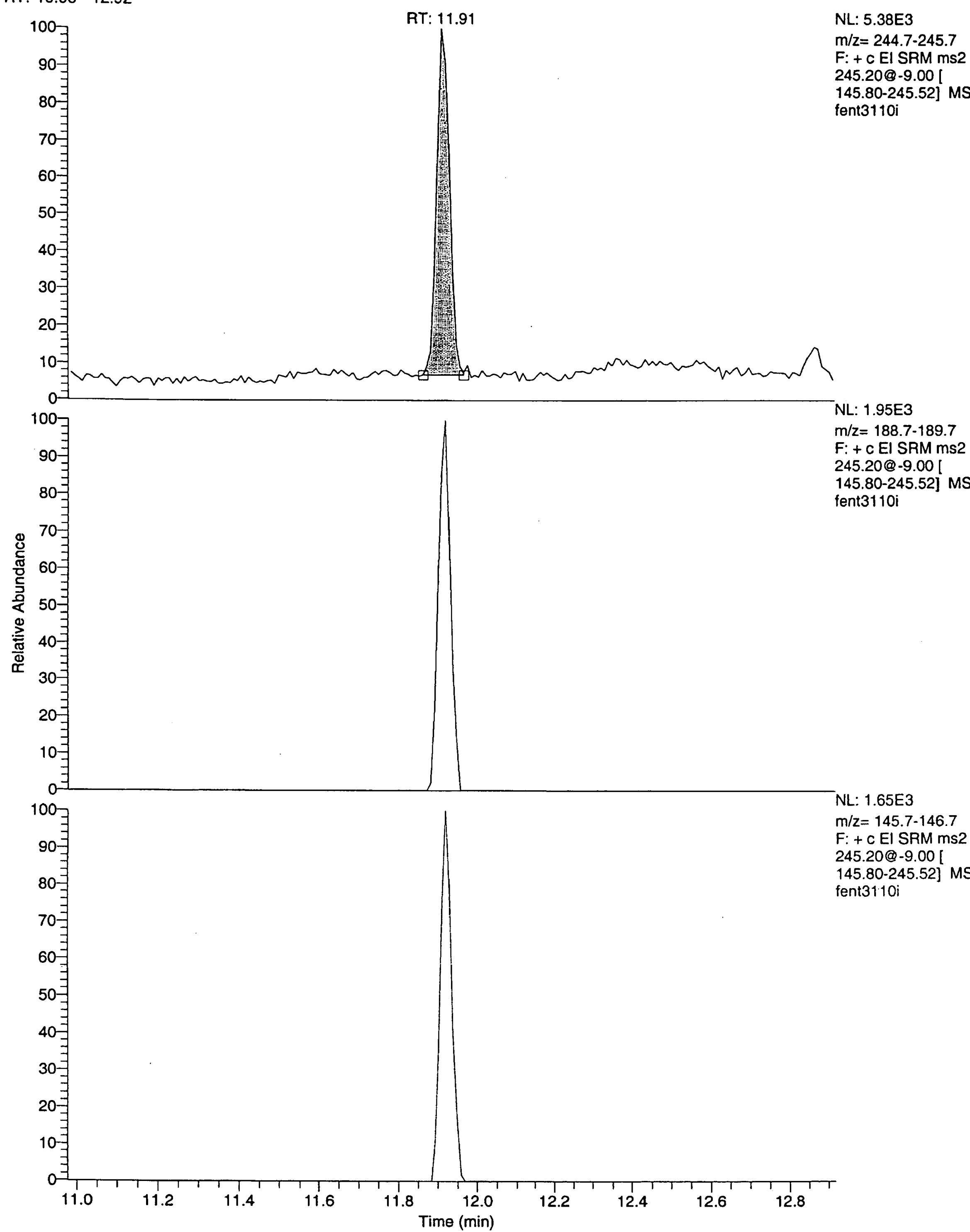

$\mathrm{NL}: 1.65 \mathrm{E} 3$

$\mathrm{m} / \mathrm{z}=145.7-146.7$ $\mathrm{F}:+\mathrm{c}$ El SRM ms2 245.20@-9.00 [ 145.80-245.52] MS fent3110i

$\mathrm{m} / \mathrm{z}=244.7-245.7$

$\mathrm{F}:+\mathrm{C}$ El SRM ms2 45.20@-9.00[ 145.80-245.52] MS ent3110i

Figure 3 : Chromatogramme obtenu par CPG/SM/SM à partir de l'échantillon de cheveux prélevé. Le tracé du haut représente l'ion parent $(\mathrm{m} / z 245)$ et les deux tracés du bas les ions fils $(\mathrm{m} / z 189$ et 146$)$ issus de la fragmentation de l'ion parent. 


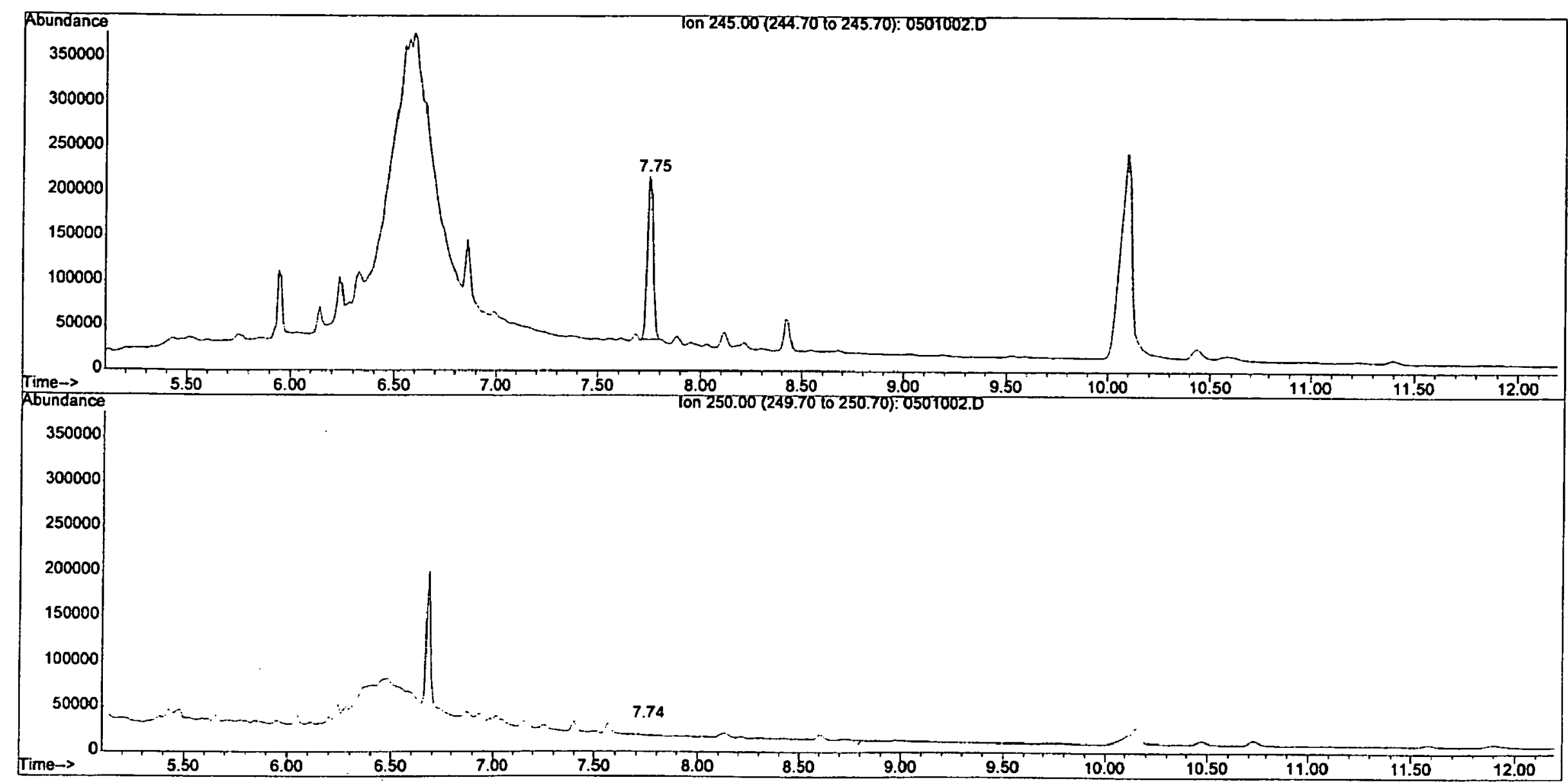

Figure 4: Chromatogramme obtenu par CPG/SM à partir de l'échantillon de cheveux prélevé.

L'analyse des cheveux permet donc de mettre en évidence des résultats urinaires négatifs suite à une abstinence momentanée durant les quelques jours précédant le prélèvement. Dans notre cas et pour des cas similaires, l'analyse des cheveux est un outil indispensable mis au service de la Justice.

Notre échantillon a également été injecté sur un chromatographe "HP 6890" couplé à un spectromètre de masse "HP 5973" (CPG/SM), à titre de comparaison. Le chromatogramme obtenu est présenté en figure 4. L'acquisition est réalisée en mode SIM (selected ion monitoring) sur 4 ions $(\mathrm{m} / z 245,189,146,250)$. Le temps de rétention du fentanyl est inférieur à celui observé en CPG/SM/SM : cela est dû a l'injection pulsée de l'échantillon $(4 \mu \mathrm{l})$ ainsi qu'à la température initiale du four de $150^{\circ} \mathrm{C}$ (contre $60^{\circ} \mathrm{C}$ en CPG/SM/SM). Comparé au tracé observé sur la $\mathrm{CPG} / \mathrm{SM}$ de paillasse, le spectromètre de masse tandem engendre un gain de spécificité qui abaisse considérablement le bruit de fond du signal des ions fils. C'est une particularité importante, surtout en Médecine Légale, où l'observation d'un faux positif peut avoir de graves conséquences.

\section{Conclusion}

Ce papier relate le cas d'un médecin soupconné de consommer illégalement du fentanyl. Après l'échec de plusieurs analyses urinaires, l'analyse de ses cheveux a permis de conclure à un abus chronique de ce stupéfiant.

\section{Références}

1. Maki D.G., Klein B.S., McCormick R.D., Alvarado C.J., Zilz M.A., Stolz S.M., Hassemer C.A., Gould J., Liegel A.R. Nosocomial Pseudomonas pickettii bacteremias traced to narcotic tampering : A case for selective drug screening of health care personnel. J. Am. Med. Assoc. $1991 ; 265$ : 981-986.

2. Ward C.F., Ward G.C., Saidman L.J. Drug abuse in anesthesia training programs : a survey. J. Am. Med. Assoc. 1970 through $1980 ; 250: 922-925$.

3. Rosenberg M. Drug abuse in oral and maxillofacial training programs. J. Oral Maxillofac. Surg. 44 : 458-462.

4. 1993 Physicians Desk Reference (47th Edition). Medical Economics Company. Montvale, NJ, 1993 : 1160-1164.

5. Wang W-L., Cone E., Zacny J. Immunoassay evidence for fentanyl in hair of surgery patients. Forensic Science International. $1993 ; 61: 65-72$.

6. Selavka C.M., Mason A.P., Riker D.,Crookham S. Determination of fentanyl in hair : the case of the crooked criminalistic. Journal of Forensic Sciences. 1995 ; $40: 681-685$.

7. Sachs S.,Uhl M., Hege-Scheuing G., Schneider E. Analysis of fentanyl and sufentanyl in hair by GC/MS/MS. Int. J. Leg. Med. $1996 ; 109$ : 213-215. 